\title{
Comparative Quantification of Arterial Lipid by Intravascular Photoacoustic-Ultrasound Imaging and Near-Infrared Spectroscopy-Intravascular Ultrasound
}

\author{
Ayeeshik Kole ${ }^{1,2} \cdot$ Yingchun Cao $^{2} \cdot$ Jie Hui $^{3} \cdot$ Islam A. Bolad $^{4} \cdot$ Mouhamad Alloosh $^{1} \cdot \mathrm{Ji}^{-X i n} \mathrm{Cheng}^{2,5} \cdot$ \\ Michael Sturek ${ }^{1,2}$
}

Received: 27 June 2018 / Accepted: 19 November 2018 / Published online: 28 November 2018

(C) The Author(s) 2018

\begin{abstract}
Intravascular photoacoustic-ultrasound (IVPA-US) imaging and near-infrared spectroscopy-intravascular ultrasound (NIRS-IVUS) are two hybrid modalities that detect arterial lipid, with comparison necessary to understand the relative advantages of each. We performed in vivo and ex vivo IVPA-US imaging of the iliac arteries of Ossabaw swine with metabolic syndrome (MetS) and lean swine to investigate sensitivity for early-stage atherosclerosis. We repeated imaging ex vivo with NIRS-IVUS for comparison to IVPA-US and histology. Both modalities showed significantly greater lipid in MetS vs. lean swine, but only IVPA-US localized the lipid as perivascular. To investigate late-stage atherosclerosis, we performed ex vivo IVPA-US imaging of a human coronary artery with comparison to NIRS-IVUS and histology. Two advanced fibroatheromas were identified, with agreement between IVPA-measured lipid area and NIRS-derived lipid content. As confirmed histologically, IVPA-US has sensitivity to detect lipid content similar to NIRSIVUS and provides additional depth resolution, enabling quantification and localization of lipid cores within plaques.
\end{abstract}

Keywords Atherosclerosis · Intravascular imaging · Photoacoustic imaging · Near-infrared spectroscopy $\cdot$ Lipid core plaque Perivascular adipose tissue $\cdot$ Swine $\cdot$ Human

$\begin{array}{llll}\text { Abbreviations } & & \text { IVUS } & \text { Intravascular ultrasound } \\ \text { CAD } & \text { Coronary artery disease } & \text { PVAT } & \text { Perivascular adipose tissue } \\ \text { MetS } & \text { Metabolic syndrome } & \text { LCBI } & \text { Lipid core burden index } \\ \text { H\&E } & \text { Hematoxylin and eosin } & \text { TCFA } & \text { Thin-capped fibroatheroma } \\ \text { NIRS } & \text { Near-infrared spectroscopy } & \text { maxLCBI } & \text { Maximum lipid core burden within } \\ \text { IVPA-US } & \text { Intravascular photoacoustic-ultrasound } & & \text { a } 4 \text { mm segment } \\ \text { PA } & \text { Photoacoustic } & \text { US } & \text { Ultrasound }\end{array}$

Ayeeshik Kole and Yingchun Cao contributed equally to this work.

Associate Editor Emanuele Barbato oversaw the review of this article

Electronic supplementary material The online version of this article (https://doi.org/10.1007/s12265-018-9849-2) contains supplementary material, which is available to authorized users.

Michael Sturek

msturek@iu.edu

1 Department of Cellular \& Integrative Physiology, Indiana University School of Medicine, 635 Barnhill Drive, MS 385, Indianapolis, IN 46202, USA

2 Weldon School of Biomedical Engineering, Purdue University, West Lafayette, IN 47907, USA
3 Department of Physics and Astronomy, Purdue University, West Lafayette, IN 47907, USA

4 Roudebush VA Medical Center and Krannert Institute of Cardiology, Indiana University School of Medicine, Indianapolis, IN 46202, USA

5 Department of Biomedical Engineering, Department of Electrical and Computer Engineering, Photonics Center, Boston University, Boston, MA 02215, USA 


\section{Introduction}

Over the past two decades, morphometric data from autopsy specimens have advanced our understanding of atherosclerotic plaque types and their progression to major adverse cardiovascular events [1, 2]. Evidence has shown that thin-capped fibroatheromas (TCFAs) are the plaque type most vulnerable to rupture and are defined by hallmarks of a thin fibrous cap, a large lipid-rich necrotic core, and inflammatory infiltrate [3-5]. Development of intravascular imaging tools to detect vulnerable plaques has been the focus of intense investigation, yet none have been shown to reliably identify TCFAs. Detection of vulnerable plaques will be instrumental in elucidating the mechanisms underlying lesion progression, the development of preventive and therapeutic interventions, and ultimately, reducing CAD-related morbidity and mortality [6-9].

Current intravascular imaging modalities aim to detect hallmarks of TCFAs as a marker of vulnerability. For example, near-infrared spectroscopy (NIRS) uses broadband light absorption to identify lipid-rich plaque and is complemented with intravascular ultrasound (IVUS), which has long been used independently to image vessel and plaque morphology only. The NIRS-IVUS hybrid modality has been successfully validated against histopathology to identify lipid-rich plaques, differentiate between culprit and non-culprit segments, and reasonably predict cardiovascular outcomes in CAD patients [10-14]. Yet, NIRS-IVUS is limited by lack of depth (or radial) resolution, distinguishing only where vascular lipid is circumferentially. This modality cannot provide information about the dimensions of lipid deposition, which is necessary for optimally assessing vulnerability, monitoring lesion progression, and pathophysiological involvement of arterial lipids.

Intravascular photoacoustic (IVPA) imaging has been the latest addition to the list of modalities and uses ultrasoundbased detection of optical absorption as a means of contrast. Photoacoustic (PA) imaging relies on the principle that small absorbance of light by $\mathrm{C}-\mathrm{H}$ bonds abundant in lipids cause thermoelastic expansion and generation of detectable acoustic waves. As a result, IVPA imaging benefits from both opticaland acoustic-based modalities: label-free chemical selectivity and ample penetration depth [15-18]. In IVPA catheters, the optical fiber and ultrasound (US) transducer have been miniaturized to fit and examine overlapping fields of view [19-22]. Thus, IVPA-US operates inherently as a hybrid intravascular catheter, capable of producing complementary cross-sectional images of vascular morphologic information and depth-resolved, lipid-specific compositional information via US and PA channels, respectively [23-25]. IVPA-US imaging has moved towards clinical translation, as limitations such as image acquisition speed and co-registration of channels have been addressed in recent years [20-22, 26, 27]. Yet, there remain significant limitations. For instance, while IVPAUS imaging has been shown to be feasible through blood, optimal sensitivity is still achieved with blood clearance or dilution with saline heavy water $\left(\mathrm{D}_{2} \mathrm{O}\right)[20,22]$.

As both IVPA-US and NIRS-IVUS detect the lipid-rich component contributing to a vulnerable plaque phenotype, comparison between the two hybrid modalities is warranted. Our aim was to elucidate the relative advantages and shortcomings of each modality.

Here, we demonstrate the ability of in vivo and ex vivo IVPA-US imaging to quantify increased perivascular adipose tissue (PVAT), a known mediator of early atherosclerotic plaque formation, using Ossabaw miniature swine, a previously characterized large animal model of metabolic syndrome (MetS) and dyslipidemia [28-31]. We further compare IVPA-US imaging to ex vivo NIRS-IVUS and histopathology. The ability to detect and localize arterial lipids in early atherosclerosis will provide insights into the role of PVAT in lesion progression. This mechanism involves "outside-to-inside" signaling from PVATderived adipokines (e.g., leptin) that diffuse to the vascular wall and synergize with more classical "inside-to-outside" mediators (e.g., lipoproteins) to promote atherosclerosis [28-31]. Furthermore, we show comparative quantification and localization of advanced atherosclerotic plaques in a fresh human coronary artery by ex vivo IVPA-US compared to NIRS-IVUS and histopathology. To our knowledge, this is the first comparison of IVPA-US to NIRS-IVUS imaging, and other existing modalities, such as single-modality grayscale IVUS.

\section{Materials and Methods}

\section{Animal Care and Use}

Ossabaw miniature swine $(n=3)$ were maintained to meet the characteristics of MetS for 8 months. For 7 of 8 months, they were fed a hypercaloric, atherogenic diet $(1 \mathrm{~kg} /$ day $)$ and were approximately age 16 months at euthanasia. For a lean control, a subset of swine $(n=2)$ were fed a standard chow diet $(0.7 \mathrm{~kg} /$ day $)$. Metabolic data including body weight, blood pressure, serum chemistry, and plasma lipids were collected to confirm development of MetS and dyslipidemia. Development of MetS and preclinical vascular disease in this naturally progressive animal model of human disease has been characterized extensively in previous work [32, 33].

Anesthesia was induced via intramuscular injection of telazol $(5-6 \mathrm{mg} / \mathrm{kg})$ and xylazine $(2.2 \mathrm{mg} / \mathrm{kg})$, and maintained with $2-4 \%$ isoflurane mixed with $100 \%$ oxygen.

\section{IVPA-US Imaging System and Catheter}

The high-speed IVPA-US imaging system used in this work has been described in detail elsewhere [21, 22, 27]. Briefly, a nanosecond $\mathrm{Nd}$ :YAG pumped optical parametric oscillator excitation laser source emitting at $1730 \mathrm{~nm}$ with a $2-\mathrm{kHz}$ 
repetition rate was used for photoacoustic excitation. A collinear catheter with a $42-\mathrm{MHz}$ transducer was used for all swine imaging (Fig. 1a). For human coronary artery imaging, a modified quasi-collinear probe design was used, in which angling of the transducer and reduction of reflection surfaces maintained optical and acoustic co-registration and improved US quality (supplementary Fig. S1) [22]. The laser pulse energy delivered to the tissue was controlled to a fluence of $50 \mathrm{~mJ} / \mathrm{cm}^{2}$, below the ANSI laser safety standard for of $1 \mathrm{~J} /$ $\mathrm{cm}^{2}$ at $1730 \mathrm{~nm}$ for the skin. The catheter was enclosed within a protective sheath composed of polyurethane and had an outer diameter of $1.6 \mathrm{~mm}$. For all IVPA-US imaging, the catheter sheath was flushed with deuterium oxide $\left(\mathrm{D}_{2} \mathrm{O}\right)$ due to decreased optical absorption compared to $\mathrm{H}_{2} \mathrm{O}$.

\section{In vivo Imaging: Angiography, IVUS, and IVPA-US}

A 7-Fr introducer sheath was inserted in the right femoral artery of swine and heparin was administered $(50 \mathrm{U} / \mathrm{kg})$. Next, a 7-Fr guiding catheter was advanced to access the right iliac artery, through which our 4.8-Fr, 42-MHz hybrid IVPAUS catheter was advanced $80 \mathrm{~mm}$ distally and two automated pullbacks were recorded at $0.5 \mathrm{~mm} / \mathrm{s}$ and 8 frames per second (fps) imaging speed (Fig. 1b-d). Next, a 3.2-Fr, 45-MHz single-modality grayscale IVUS catheter (Revolution, Volcano, Corp.) was advanced $80 \mathrm{~mm}$ distally and a pullback was recorded at $0.5 \mathrm{~mm} / \mathrm{s}$ and $30 \mathrm{fps}$. In one lean control animal, right and left iliac arteries were both imaged. All in vivo imaging was performed through luminal blood, without clearance or dilution. Following imaging, the animals were euthanatized by isoflurane overdose and cardiectomy.

\section{Ossabaw Swine Tissue Recovery and Preparation}

Following euthanasia, the iliac artery was isolated, marked with a suture knot $80 \mathrm{~mm}$ distal to the guiding catheter, and dissected out of the animal. Side branches and the distal end were ligated with suture for pressure-perfusion during ex vivo imaging, followed by formalin fixation.

\section{Human Tissue Recovery and Preparation}

A fresh human heart was harvested from a 59-year-old male undergoing transplant surgery, within $24 \mathrm{~h}$ of explant. The patient had coronary heart disease risk factors of smoking, hypertension, and obesity. The right coronary artery was excised, the ostium was cannulated with a 6-Fr introducer sheath, and side branches and the distal end of the artery were ligated with suture for pressure-perfusion. Artery anatomy was observed by ex vivo angiography. All imaging was performed within $48 \mathrm{~h}$ of dissection.

\section{Ex vivo Imaging: IVPA-US and NIRS-IVUS}

The hybrid IVPA-US catheter was advanced to the $80-\mathrm{mm}$ mark of Ossabaw iliac artery segments and perfused with
Fig. 1 IVPA-US imaging probe and in vivo imaging procedure. a Schematic of the IVPA-US collinear catheter design, in which the acoustic and optical paths overlap, after a series of reflections off the rod mirror and optical fiber surfaces. $\mathbf{b}$ Schematic of the in vivo procedure, in which the IVPA-US catheter was advanced $80 \mathrm{~mm}$ into the iliac artery of swine. c Angiogram with contrast to visualize vascular anatomy. $\mathbf{d}$ Angiogram without contrast to confirm IVPA-US catheter placement at $80 \mathrm{~mm}$ distal to the introducer sheath a
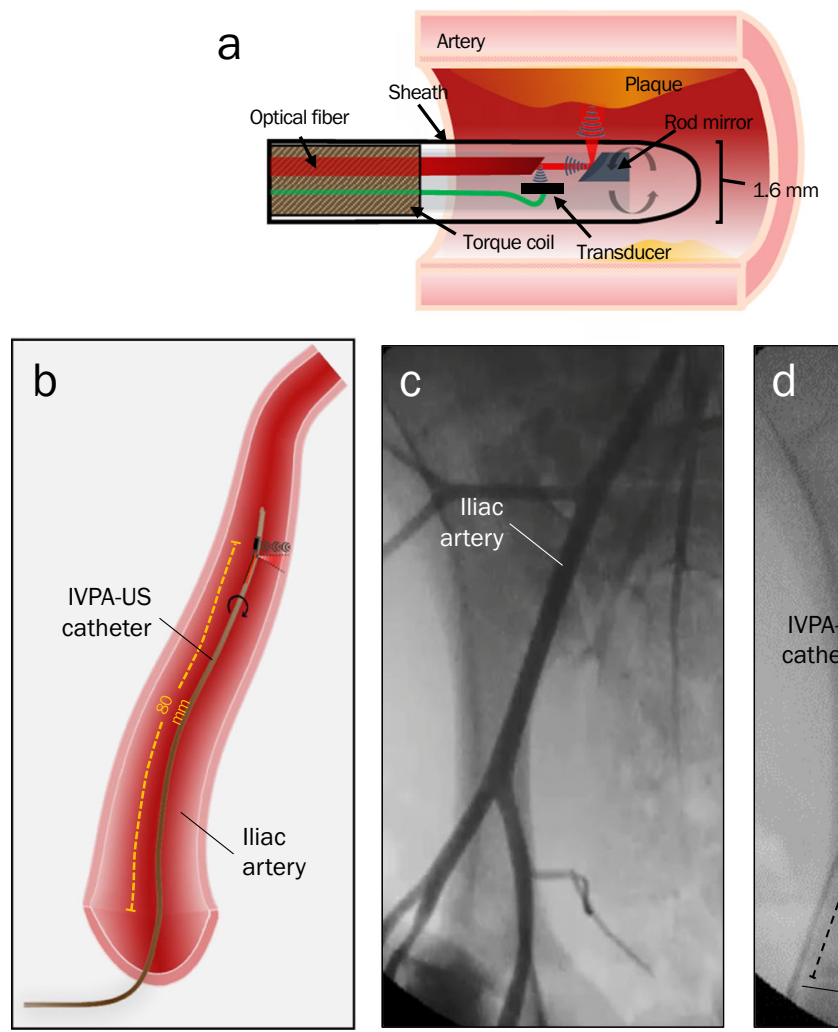

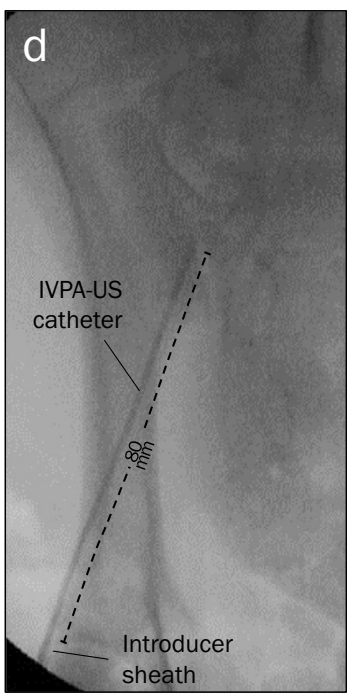


$1 \mathrm{X}$ phosphate buffered saline at approximately $70 \mathrm{~mL} / \mathrm{min}$, during which an automated pullback was recorded at $0.25 \mathrm{~mm} / \mathrm{s}$ and $4 \mathrm{fps}$ imaging speed. This procedure was repeated with a $3.2-\mathrm{Fr}, 40-\mathrm{MHz}$ hybrid NIRS-IVUS catheter (TVC Insight, Infraredx, Inc.) with a $0.5-\mathrm{mm} / \mathrm{s}$ pullback speed and $16 \mathrm{fps}$ imaging speed. Human coronary artery imaging was performed following the same protocol, with the pullbacks starting approximately $70 \mathrm{~mm}$ distal to the introducer sheath.

\section{IVPA-US and NIRS-IVUS Quantitative Analysis}

To compare pullbacks from two hybrid modalities collected with different optimal frame rates and pullback speeds, we used angiography and landmarks, such as the introducer sheath and fiduciary side branches, to align frames.

IVPA-US imaging data were post-processed to reduce noise and pixels at which PA intensity was greater than a set threshold were counted per cross-sectional frame to calculate lipid area (four times the background noise for all ex vivo data sets and five times the background noise for all in vivo data sets). Thresholds were determined by an investigator blind to sample group, other imaging results, and histology. For human coronary artery imaging, the lipid core volume was calculated per lesion within a 4-mm segment by identifying depth and angle boundaries within which pixels were counted. All lipid area measurements were calculated using MATLAB software (MathWorks, Inc.).

Cross-sectional lipid areas were binned and averaged for correlation analysis between repeat in vivo and ex vivo pullbacks to assess reproducibility of data.

The NIRS-IVUS TVC Insight system was used to generate two-dimensional "chemograms" representing probability of the presence of lipid by a yellow color, with longitudinal pullback position on the $x$-axis and circumferential position on the $y$-axis. As NIRS-IVUS spectral depth is $1 \mathrm{~mm}$ or less, lipid area was estimated by measuring the yellow-colored regions on the 2D chemograms. The system also generated "block chemograms," representing the artery in 2-mm segments with discrete colorized representation of the probability of lipid present. We defined blocks colored red as having no lipid present $(p<0.57)$ and all other colors as having increased probability of lipid present [34]. The total lipid core burden index (LCBI) and maximum LCBI within a 4-mm segment $\left(\operatorname{maxLCBI}_{4 \mathrm{~mm}}\right)$ were also calculated by the system.

Calcification was measured from the IVUS channel of the hybrid modalities and defined as strong echogenicity with acoustic shadowing. The total length was calculated as continuous frames in which calcification was present and maximum arc was calculated as the largest angle of acoustic shadowing. All analysis of the IVUS channel was performed using ImageJ software (National Institutes of Health).

\section{Histological Preparation and Analysis}

Imaged arteries were pressure-fixed using $10 \% \mathrm{w} / \mathrm{v}$ formalin and placed in formalin overnight. Contralateral control iliac arteries from Ossabaw swine were not pressure-fixed. Arteries were then grossly sectioned in 3- to 4-mm segments. Next, all segments were paraffin embedded, thin sectioned, and stained with hematoxylin and eosin (H\&E) and Verhoeff-Van Gieson elastin stain. In the human coronary artery with advanced atherosclerosis, Russel-Movat's pentachrome stain was used to identify and characterize lipid core plaques [1, 10]. Lipid-rich necrotic cores were defined as morphologically distinct empty and/or clear, granular, mostly anucleate spaces, defined as the light-microscopic characteristics of lipid gruel, with macrophage infiltration, cholesterol clefts, or calcification $[1,2,35]$. Necrotic core area was calculated using ImageJ software.

\section{Statistics}

All data are described as mean \pm SEM. Statistical significance was set a priori at $p<0.05$. Unpaired, two-tailed Student's $t$ tests, Fisher's exact test, and Pearson's correlation were used as appropriate using GraphPad Prism statistical software (GraphPad Software, Inc.).

\section{Results}

\section{IVPA-US Sensitivity for Early-Stage Atherosclerosis in Ossabaw Swine}

Ossabaw swine on hypercaloric, atherogenic diet developed the characteristics of MetS, including significantly greater total cholesterol, triglycerides, and weight at sacrifice compared to swine in the lean control group (Table 1). Despite the development of MetS in swine, on in vivo and ex vivo iliac artery imaging of both groups, no significant intimal lipid deposition was detected on all modalities, consistent with histology which showed mild vascular disease evident as minimal neointimal hyperplasia (Fig. 2).

Table 1 Metabolic characteristics of Ossabaw swine

\begin{tabular}{llll}
\hline & Lean $(n=2)$ & $\operatorname{MetS}(n=3)$ & $p$ value \\
\hline Sex (M/F) & $1 / 1$ & $0 / 3$ & N/A \\
Weight at sacrifice (kg) & $50.5 \pm 10.5$ & $95.0 \pm 1.5^{*}$ & 0.01 \\
Mean weight gain (kg) & 31.0 & $58.7 \pm 3.0$ & N/A \\
Plasmalipids & & & \\
Cholesterol (mg/dL) & $71 \pm 12$ & $465 \pm 53^{*}$ & 0.01 \\
Triglycerides (mg/dL) & $26 \pm 2$ & $63 \pm 4^{*}$ & 0.01 \\
\hline
\end{tabular}

$* p<0.05$ 

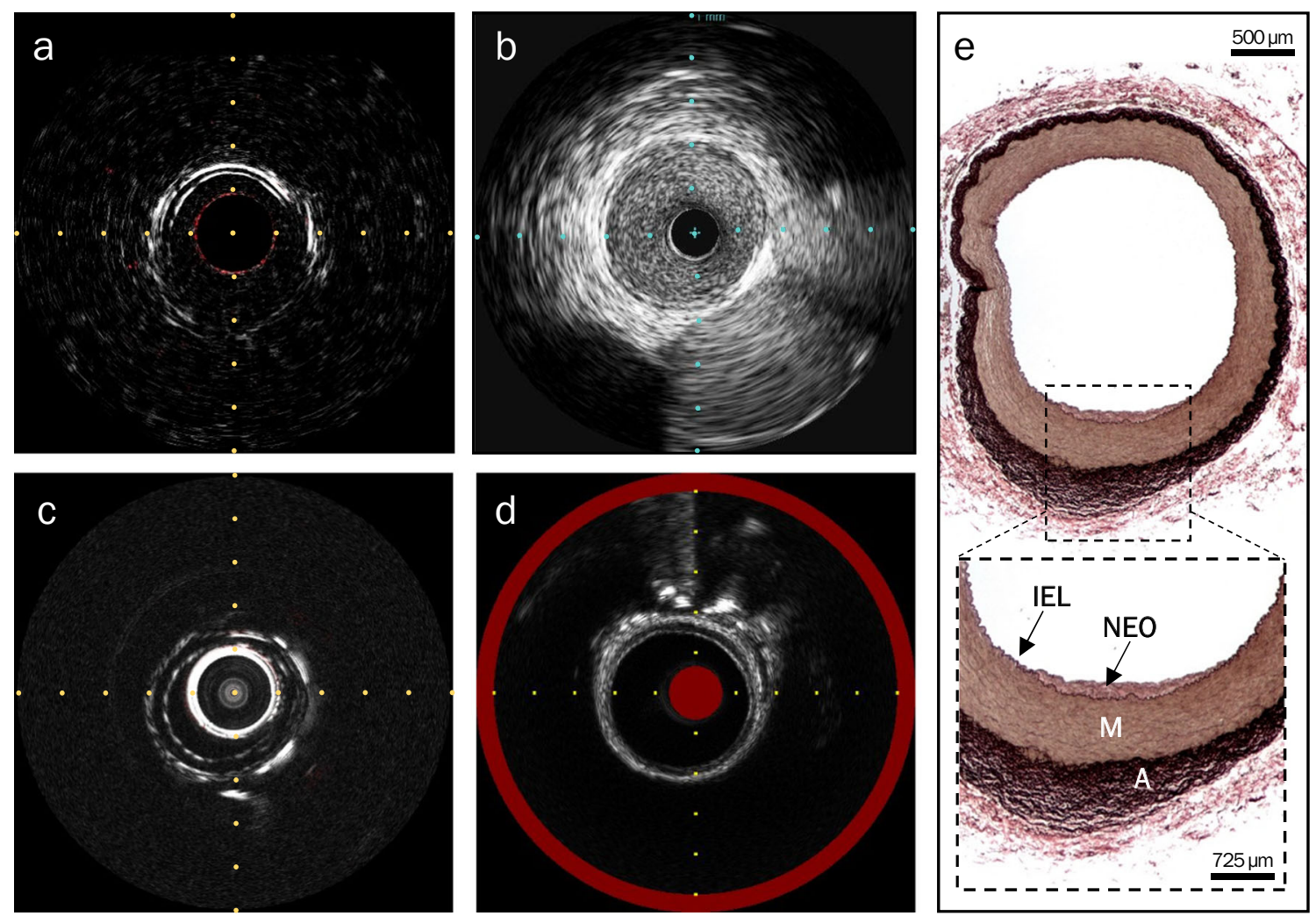

Fig. 2 Representative imaging and histology from a MetS Ossabaw swine iliac artery showing no intimal lipid deposition and only early-stage neointimal thickening. a In vivo IVPA-US still frame, in which no lipid deposition (absence of red) and mild neointimal hyperplasia (three-layer appearance, grayscale IVUS channel) was observed. b In vivo singlemodality grayscale IVUS still frame, depicting the three-layer appearance. c Ex vivo IVPA-US still frame, in which no lipid deposition and the three-

layer appearance were observed. d Ex vivo NIRS-IVUS still frame, in which no lipid deposition (absence of yellow circumferentially) and the three-layer appearance (grayscale IVUS channel) were observed. e Verhoeff-Van Gieson stained histological section, showing typical artery morphology with a small area of neointimal thickening (NEO) on the luminal side of the internal elastic lamina (IEL). M media, A adventitia. Horizontal and vertical axis tracings are $1 \mathrm{~mm}$ apart

However, in specific segments of the iliac arteries, low levels of lipid signal were detected by both IVPA-IVUS and NIRS-IVUS, which were further localized to the perivascular region and confirmed as PVAT by histology (Fig. 3). Similar signal was detected by NIRS-IVUS at corresponding regions of interest, albeit without localization of the lipid depot radially due to lack of depth resolution (Fig. 3d).

On in vivo IVPA imaging, we found that the average crosssectional lipid area was significantly greater in the iliac arteries of swine with MetS as compared to lean swine $(0.089 \pm$ $0.016 \mathrm{~mm}^{2}$ vs. $0.059 \pm 0.001 \mathrm{~mm}^{2} ; p<0.0001$ ) (Fig. 4a). These results were also observed on ex vivo imaging $(0.032$ $\pm 0.001 \mathrm{~mm}^{2}$ vs. $0.005 \pm 0.0002 \mathrm{~mm}^{2} ; p<0.0001$ ) (Fig. 4b).

\section{Correlation of IVPA-US with NIRS-IVUS in Early-Stage Atherosclerosis}

On ex vivo NIRS-IVUS imaging, we found similar results to IVPA-US imaging, as arteries of swine with MetS had significantly more 2 -mm block chemograms with increased probability of lipid present than arteries of lean swine (8/117 blocks with lipid vs. $0 / 80$ blocks with lipid; $p=0.02$ ). Taken together, these results conclude IVPA-US is comparable to NIRS-IVUS in sensitivity for the detection of increased lipid content. Furthermore, depth resolution of IVPA-US imaging allowed localization of lipid deposition to PVAT, a known mediator of early atherogenesis $[28-31]$.

\section{IVPA-US Imaging In vivo Safety and Reproducibility}

We evaluated the safety of the in vivo imaging procedure and reproducibility of our results. On angiography, we did not observe significant vasospasm after IVPA-US pullback (supplementary Fig. S2a-b). On histology, we did not observe any significant damage to the internal elastic lamina of imaged arteries compared to contralateral control arteries (supplementary Fig. S2c-f). Additionally, in three swine iliac arteries, we performed repeat in vivo pullbacks and there was significant correlation of lipid area between pullbacks ( $p<0.0001$ for all data sets) (supplementary Fig. S3). In a MetS-group swine iliac artery, we further compared lipid area measures from in vivo pullbacks with ex vivo pullback data and we also found significant correlation ( $p<0.05$ for all data sets) (supplementary Fig. S4). 

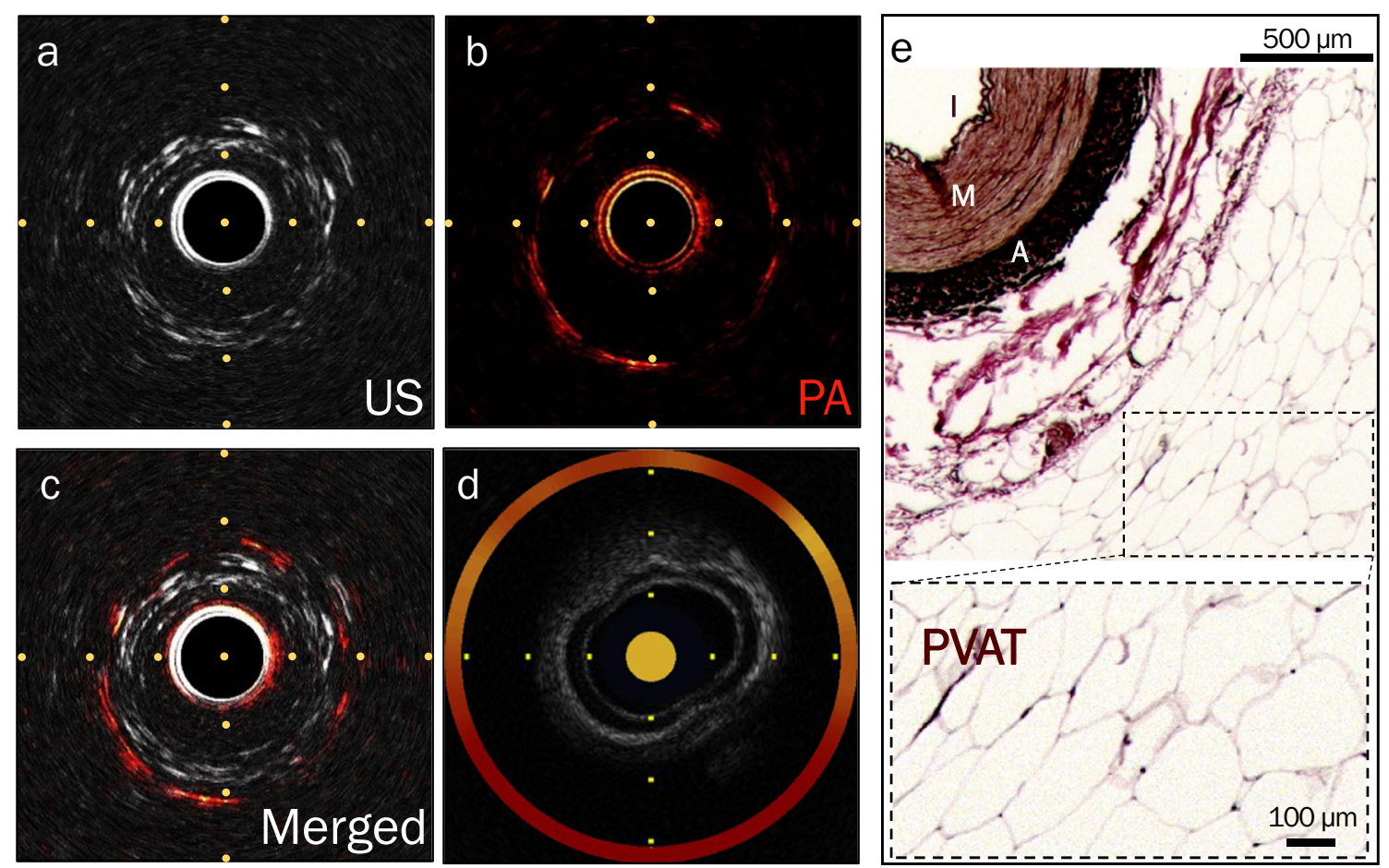

Fig. 3 Representative in vivo IVPA-US imaging from a MetS Ossabaw swine iliac artery showing a lipid signal by both modalities. a Unmerged IVUS channel still frame showing a three-layer appearance of the iliac artery. b Unmerged IVPA channel still frame showing circumferential distribution and depth of lipid signal (red). c Merged composite showing depth-resolved lipid signal localized to the perivascular region. d NIRS-IVUS still frame showing grayscale IVUS channel and lipid

\section{IVPA-US Sensitivity for Late-Stage Atherosclerosis in a Human Coronary Artery}

On ex vivo imaging of a fresh human right coronary with atherosclerosis, we identified two advanced fibroatheromas with calcification on both IVPA-US and NIRS-IVUS (Fig. 5). We defined the atheroma proximal to the introducer sheath as lesion 1 and the distal atheroma as lesion 2 (Table 2). On both hybrid modalities, calcification was evident on

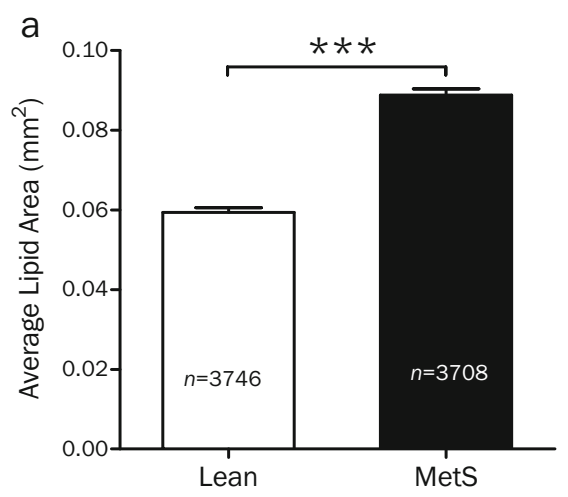

Fig. 4 Average cross-sectional lipid area in iliac arteries as determined by IVPA-US imaging. The average cross-sectional lipid area, as calculated by photoacoustic signal intensity per frame above a pre-determined signal (yellow) circumferentially, but not the precise radial depth into the arterial wall and perivascular regions. e Verhoeff-Van Gieson stained histological section showing abundant perivascular adipose tissue (PVAT, inset), noted by the presence of round and empty adipocytes. I intima, M media, A adventitia. Horizontal and vertical axis tracings are $1 \mathrm{~mm}$ apart

ultrasound by strong echogenicity with acoustic shadowing (Fig. 5b, c, panels III and IV). Furthermore, lipid was present as indicated by yellow color on the circumferential chemogram by NIRS-IVUS and red color on cross-sectional images by IVPA-US. Characterization of the atherosclerotic features in both lesions were confirmed by histopathology (Fig. 3d).

On IVPA-US imaging, we calculated the average crosssectional lipid area within a 4-mm segment for both lesions. Lesion 1 had an average lipid area of $0.15 \pm 0.004 \mathrm{~mm}^{2}$ (range

b

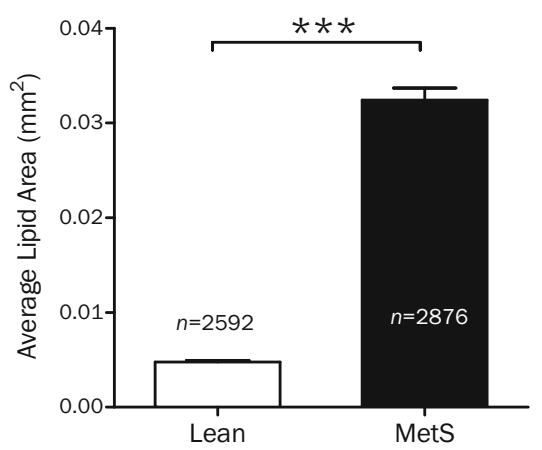

threshold, is significantly greater in the iliac arteries of swine with MetS as compared to lean swine, both by in vivo (a) and ex vivo (b) imaging; *** $p<0.0001 ; n$ refers to number of cross-sectional frames analyzed 

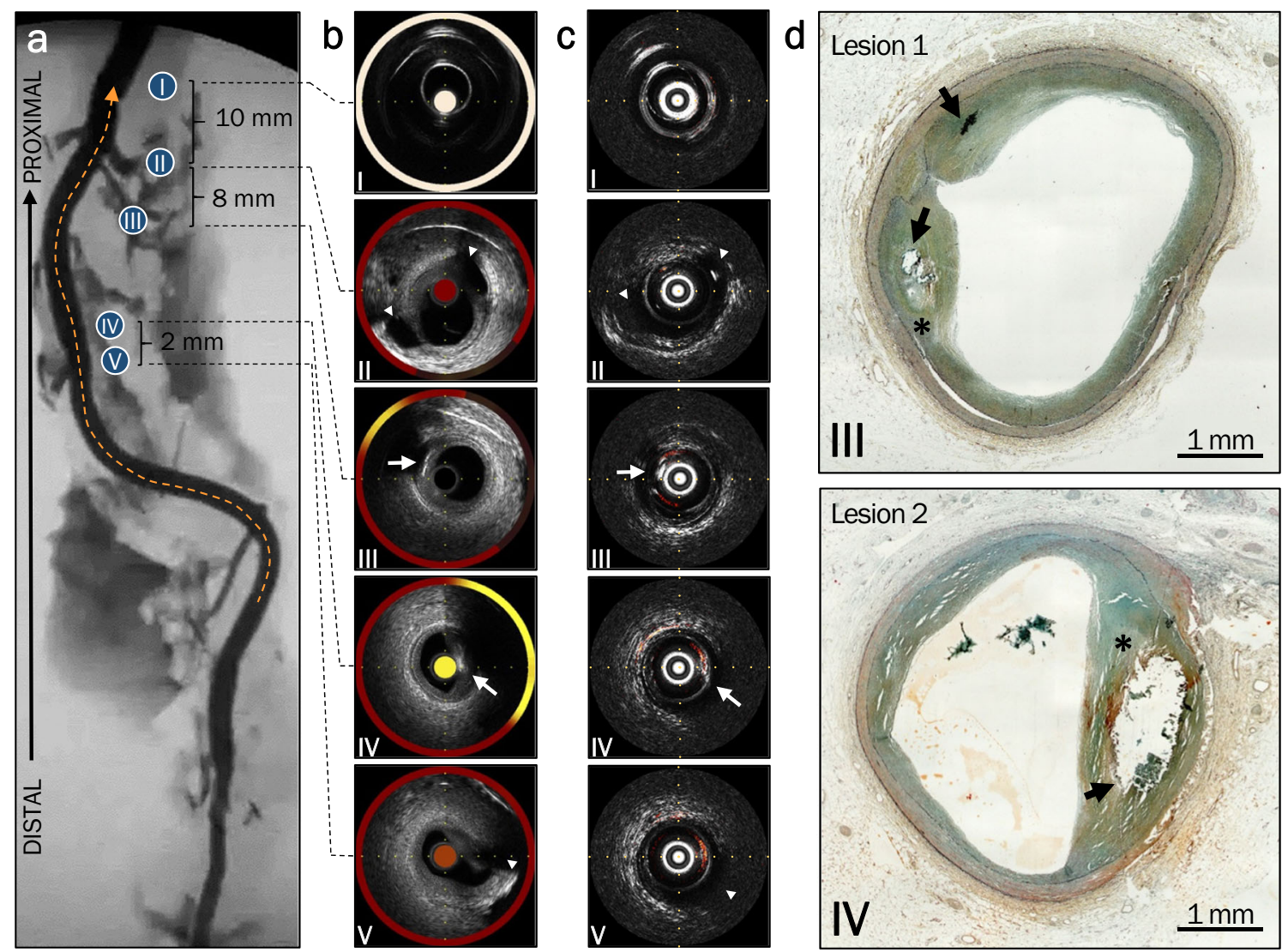

Fig. 5 Correlation between modalities in a fresh human coronary artery. a Ex vivo angiogram of the artery, in which the sheath (I), side branches (II, V), and lesions locations (III, IV) are identified. Location III refers to Lesion 1 and location IV to Lesion 2. b, c Corresponding crosssectional still frames are shown from NIRS-IVUS (b) in which lipid is

$0.08-0.24)$ and lesion 2 had an average lipid area of $0.40 \pm$ $0.03 \mathrm{~mm}^{2}$ (range 0.04-1.04). Particularly, lesion 2, within the measured 4-mm segment, had a significantly greater average lipid area than the remaining length of artery $\left(0.40 \mathrm{~mm}^{2}\right.$ vs. $\left.0.26 \mathrm{~mm}^{2} ; p<0.0001\right)$. We further measured the total lipid area within a $4-\mathrm{mm}$ segment for each lesion (lesion 1 $9.57 \mathrm{~mm}^{2}$, lesion $225.95 \mathrm{~mm}^{2}$ ).

Lastly, depth resolution and co-registered imaging allowed us to identify the contiguous lipid areas adjacent to calcification as the plaque lipid core and calculated the volumes as $0.11 \mathrm{~mm}^{3}$ and $0.25 \mathrm{~mm}^{3}$ for lesions 1 and 2, respectively. Relation of the plaque lipid core to calcification and the lumen border is further illustrated by three-dimensional reconstruction of the 4-mm segments (supplementary Videos 1 and 2).

\section{Correlation of IVPA-US with NIRS-IVUS and Histology in Late-Stage Atherosclerosis}

Quantifications by IVPA-US imaging were in agreement with similar measures made by NIRS-IVUS imaging and histopathology. On NIRS-IVUS, we found lesion 2 to have a high maxLCBI ${ }_{4 \mathrm{~mm}}$ value of 326 , consistent with shown with yellow and from IVPA-US (c) in which lipid is represented by red. Notable side branches are indicated by arrowheads and calcification by arrows. d Movat's pentachrome stained histological sections of lipid core lesions $(*)$ with calcification (arrows). Horizontal and vertical axis tracings are $1 \mathrm{~mm}$ apart

the large lipid area and core volume measured by IVPAUS. No maxLCBI ${ }_{4 \mathrm{~mm}}$ was calculated for lesion 1 due to lack of sufficient spectrally valid pixels. Using the generated chemogram, we approximated lipid areas as $5.22 \mathrm{~mm}^{2}$ for lesion 1 and $26.38 \mathrm{~mm}^{2}$ for lesions 2 , comparable to the values measured by IVPA-US.

In addition, we characterized the calcification in both lesions by measuring the total length and maximal arc of calcification, as shown in Table 2. Both IVPA-US and NIRS-IVUS systems showed similar values, demonstrating comparable resolution of the US channel for the identification of calcification.

Lastly, on histopathology we measured histologic necrotic core areas of $0.42 \mathrm{~mm}^{2}$ and $1.36 \mathrm{~mm}^{2}$ for lesions 1 and 2 , respectively. The larger necrotic core area on histopathology compared to the average cross-sectional lipid area by IVPAUS was anticipated, as necrotic cores contained features such as prominent calcification, in addition to lipid.

Comparative imaging by two hybrid modalities and goldstandard histopathology confirm that depth-resolved IVPAUS has the capability to quantify and localize plaque lipid cores with calcification. 
Table 2 Comparison of lesion characteristics from ex vivo imaging of a human right coronary artery by NIRS-IVUS and IVPA-US

\begin{tabular}{|c|c|c|}
\hline & Lesion 1 (proximal) & Lesion 2 (distal) \\
\hline \multicolumn{3}{|c|}{ NIRS-IVUS measured lesion characteristics } \\
\hline $\operatorname{maxLCBI}{ }_{4 \mathrm{~mm}}$ & $\mathrm{~N} / \mathrm{A}$ & 326 \\
\hline Total lipid area $\left(\mathrm{mm}^{2}\right)$ & 5.22 & 26.38 \\
\hline Total calcified length (mm) & 3.1 & 2.0 \\
\hline Maximum arc of calcification $\left({ }^{\circ}\right)$ & 46.2 & 66.7 \\
\hline \multicolumn{3}{|c|}{ IVPA-US measured lesion characteristics } \\
\hline Average lipid area $\mathrm{mmm}_{4 \mathrm{~m}}\left(\mathrm{~mm}^{2}\right)$ & $0.15 \pm 0.004$ & $0.40 \pm 0.03$ \\
\hline Total lipid area $a_{4 \mathrm{~mm}}\left(\mathrm{~mm}^{2}\right)$ & 9.57 & 25.95 \\
\hline Lesion lipid core volume $\left(\mathrm{mm}^{3}\right)$ & 0.11 & 0.25 \\
\hline Total calcified length (mm) & 3.4 & 2.4 \\
\hline Maximum arc of calcification $\left({ }^{\circ}\right)$ & 58.1 & 66.4 \\
\hline
\end{tabular}

\section{Discussion}

Previous studies using NIRS-IVUS clearly show the clinical value of identification of arterial lipid as a prognostic indicator of cardiovascular outcomes [12-14]. Those preliminary studies support the notion that accurate detection of arterial lipid content by a reliable intravascular imaging modality, or combination of multiple modalities, could be predictive of lesions at high risk to progress to cardiovascular events. Multiple prospective studies, including PROSPECT II ABSORB sub-study (NCT02171065) and the Lipid-Rich Plaque Study (NCT02033694), aim to answer this important question [36]. As the only FDA-approved device for measuring lipid-rich plaques, NIRS-IVUS undoubtedly has great promise in clinical practice. Yet, lack of depth resolution limits quantitative imaging of lipid and relies upon LCBI as a derived measure to distinguish between high-risk and low-risk plaques. In other words, NIRS-IVUS provides an excellent biochemical assay of bulk lipid content in sections of artery, but the precise location of lipid in the vascular wall and perivascular compartment is not provided.

Here, we conducted the first head-to-head comparison of IVPA-US to NIRS-IVUS and highlight the value of depth resolution in lipid detection. We have demonstrated the capability of IVPA-US to detect both superficial and deep lipid, i.e., lipid within an advanced plaque core and perivascular lipid of early-stage atherosclerosis.

By ex vivo imaging of a human coronary artery, we identified advanced lipid core plaques by both NIRS-IVUS and IVPA-US. We were able to quantify and localize the lipid at corresponding lesions using IVPA-US only, by virtue of depth resolution with co-registration of the two modalities. A demonstrated advantage with depth-resolved lipid imaging is volumetric quantification of lipid plaque cores, which would permit more accurate longitudinal studies monitoring plaque progression and regression. Alterations in plaque morphology and composition could be more meaningful with visualization of lipid core dimensions and relation to other plaque components (i.e., fibrous cap, calcification). This would aid in elucidation of the prevalence and mechanisms of plaque development, stability, and rupture [37].

Additionally, by in vivo IVPA-US imaging of iliac arteries, we detected early-stage atherosclerotic changes in the form of increased PVAT in Ossabaw swine with MetS versus lean swine. Intravascular catheter detection of PVAT, although not considered vulnerable pathology, would be a valuable measure to investigate its role in early atherogenesis. Many groups have observed an enhanced pro-inflammatory milieu in adipose tissue of patients with CAD versus those without disease, supporting the hypothesis that PVAT exerts paracrine stimulation of atherogenesis in an "outside-to-inside" manner [28, 38]. In crosssectional clinical studies, PVAT quantity is strongly associated with several atherosclerotic measures, including plaque presence, percent coronary artery stenosis, coronary artery calcium score, and future major adverse cardiovascular events [29, 30]. Lastly, surgical resection of local coronary PVAT in swine models has been shown to attenuate underlying coronary atherosclerosis, providing the first causal evidence [31]. However, PVAT is traditionally measured by computed tomography along the cardiac short axis and co-localization to intravascular measures such as plaque burden are not possible, limiting the ability to assess the causal relationship. In other words, the field currently does not have the tools necessary to answer the question: do local depots of PVAT precede the development of underlying atherosclerosis? This shortcoming can be addressed by IVPAUS, which has penetration depths of up to $6 \mathrm{~mm}$ and can image plaque burden and PVAT in one procedure, enabling needed longitudinal natural history studies [21].

Lastly, clinical translation of IVPA-US will require validation against a robust data set of human histopathology conducted ex vivo [10]. Here, we have shown evidence in swine demonstrating correlation between ex vivo imaging through saline and in vivo imaging through blood. Additionally, we have confirmed safety of our in vivo procedure, in which there were no peri-procedural complications. Lastly, we observed no irritation to the artery as indicated by insignificant post-pullback vasospasm and no disruption to the internal elastic lamina. 
This study is limited by the lack of intimal lipid in the iliac arteries of Ossabaw swine and subsequent absence of analysis for correlation of in vivo neointimal lipid imaging to histopathology. The observation of increased PVAT depots, no neointimal lipid, and mild neointimal thickening is very intriguing and could provide new insights about atherogenesis. In future studies, we intend to employ longer duration MetS phenotypic development and longitudinal IVPA-US imaging to determine whether increased PVAT precedes an increase in neointimal lipid. We will also address catheter size and flexibility limitations to image coronary arteries, in which atherosclerotic disease develops more predictably [32].

Lastly, our human artery sample size is small and does not include a wide range of atherosclerotic plaque phenotypes. Thus, we were unable to calibrate our thresholding algorithm for IVPAderived lipid area to correlate with histopathological lipid-rich necrotic core area. We intend to build a larger calibration data set to address this limitation. In addition, we anticipate future application of spectral algorithms to differentiate between cholesterol subtypes, as a surrogate marker of inflammation [39].

\section{Conclusion}

As intravascular imaging modalities for lipid imaging advance in capability and combination with others, comparison between systems will be instrumental in determining the relative advantages and disadvantages of each. We have demonstrated detection of increased lipid deposition in Ossabaw swine with earlystage atherosclerosis versus lean swine by IVPA-US imaging, in agreement with NIRS-IVUS imaging of the same specimens. Further, we have shown detection of late-stage atherosclerotic plaques in human coronary arteries by both modalities. In conducting this comparison, we reveal the advantage of depthresolved IVPA-US imaging in varying stages of atherosclerosis for the localization and quantification of both superficial and deep lipid content, even extending into the perivascular region.

Acknowledgements The authors acknowledge Jim Byrd, Jonathan Lenkey, Sam Robertson, and Sarah Davis for assistance with in vivo imaging and histology analysis.

Funding This study was funded by the R01HL125385 to J.-X. Cheng and M. Sturek, Center of Excellence in Cardiovascular Research Grant and Fortune-Fry Ultrasound Research Fund to M. Sturek, American Heart Association Postdoctoral Fellowship 16POST27480018 to Y. Cao, and Hugh W. and Edna M. Donnan Fellowship to A. Kole.

\section{Compliance with Ethical Standards}

Conflict of Interest The authors declare that they have no conflicts of interest.

Ethical Approval All applicable international, national, and/or institutional guidelines for the care and use of animals were followed. The protocols were approved by the Institutional Animal Care and Use Committee at the Indiana University School of Medicine.

Human Subjects This article does not contain any studies with human participants performed by any of the authors, as approved by exemption, per the use of discarded human tissue, by the Indiana University Institutional Review Board.

Clinical Relevance IVPA-US is an emerging catheter-based hybrid imaging technique capable of quantitative localization of arterial lipid in early- to late-stage atherosclerosis. This unique capability permits future longitudinal studies to monitor progression to vulnerable plaque phenotypes and the associated mechanisms of rupture leading to acute coronary syndrome, a leading cause of morbidity and mortality.

Open Access This article is distributed under the terms of the Creative Commons Attribution 4.0 International License (http:// creativecommons.org/licenses/by/4.0/), which permits unrestricted use, distribution, and reproduction in any medium, provided you give appropriate credit to the original author(s) and the source, provide a link to the Creative Commons license, and indicate if changes were made.

\section{References}

1. Virmani, R., Kolodgie, F. D., Burke, A. P., Farb, A., \& Schwartz, S. M. (2000). Lessons from sudden coronary death: a comprehensive morphological classification scheme for atherosclerotic lesions. Arteriosclerosis, Thrombosis, and Vascular Biology, 20(5), 1262-1275.

2. Yahagi, K., Kolodgie, F. D., Otsuka, F., Finn, A. V., Davis, H. R., Joner, M., et al. (2016). Pathophysiology of native coronary, vein graft, and in-stent atherosclerosis. Nature Reviews. Cardiology, 13(2), 79-98. https://doi.org/10.1038/nrcardio.2015.164.

3. Finn, A. V., Nakano, M., Narula, J., Kolodgie, F. D., \& Virmani, R. (2010). Concept of vulnerable/unstable plaque. Arteriosclerosis, Thrombosis, and Vascular Biology, 30(7), 1282-1292. https://doi. org/10.1161/atvbaha.108.179739.

4. Virmani, R., Burke, A. P., Farb, A., \& Kolodgie, F. D. (2006). Pathology of the vulnerable plaque. Journal of the American College of Cardiology, 47(8 Suppl), C13-C18. https://doi.org/10. 1016/j.jacc.2005.10.065.

5. Kolodgie, F. D., Burke, A. P., Farb, A., Gold, H. K., Yuan, J., Narula, J., et al. (2001). The thin-cap fibroatheroma: a type of vulnerable plaque: the major precursor lesion to acute coronary syndromes. Current Opinion in Cardiology, 16(5), 285-292.

6. Virmani, R. (2011). Are our tools for the identification of TCFA ready and do we know them? JACC Cardiovasc Imaging, 4(6), 656-658. https://doi.org/10.1016/j.jcmg.2011.01.019.

7. Fleg, J. L., Stone, G. W., Fayad, Z. A., Granada, J. F., Hatsukami, T. S., Kolodgie, F. D., et al. (2012). Detection of high-risk atherosclerotic plaque: report of the NHLBI Working Group on current status and future directions. JACC Cardiovasc Imaging, 5(9), 941-955. https://doi.org/10.1016/j.jcmg.2012.07.007.

8. Puri, R., Tuzcu, E. M., Nissen, S. E., \& Nicholls, S. J. (2013). Exploring coronary atherosclerosis with intravascular imaging. International Journal of Cardiology, 168(2), 670-679. https://doi. org/10.1016/j.ijcard.2013.03.024.

9. Bourantas, C. V., Jaffer, F. A., Gijsen, F. J., van Soest, G., Madden, S. P., Courtney, B. K., et al. (2016). Hybrid intravascular imaging: recent advances, technical considerations, and current applications in the study of plaque pathophysiology. European Heart Journal, 38(6), 400-412. https://doi.org/10.1093/eurheartj/ehw097.

10. Gardner, C. M., Tan, H., Hull, E. L., Lisauskas, J. B., Sum, S. T., Meese, T. M., et al. (2008). Detection of lipid core coronary plaques 
in autopsy specimens with a novel catheter-based near-infrared spectroscopy system. JACC: Cardiovascular Imaging, 1(5), 638648. https://doi.org/10.1016/j.jcmg.2008.06.001

11. Madder, R. D., Puri, R., Muller, J. E., Harnek, J., Gotberg, M., VanOosterhout, S., et al. (2016). Confirmation of the intracoronary near-infrared spectroscopy threshold of lipid-rich plaques that underlie ST-segment-elevation myocardial infarction. Arterioscler Thromb Vasc Biol. https://doi.org/10.1161/ATVBAHA.115.306849.

12. Madder, R. D., Husaini, M., Davis, A. T., VanOosterhout, S., Khan, M., Wohns, D., et al. (2016). Large lipid-rich coronary plaques detected by near-infrared spectroscopy at non-stented sites in the target artery identify patients likely to experience future major adverse cardiovascular events. European Heart Journal Cardiovascular Imaging, 17(4), 393-399. https://doi.org/10.1093/ehjci/jev340.

13. Danek, B. A., Karatasakis, A., Karacsonyi, J., Alame, A., Resendes, E., Kalsaria, P., et al. (2017). Long-term follow-up after nearinfrared spectroscopy coronary imaging: Insights from the lipid cORe plaque association with CLinical events (ORACLE-NIRS) registry. Cardiovascular Revascularization Medicine, 18(3), 177181. https://doi.org/10.1016/j.carrev.2016.12.006.

14. Schuurman, A. S., Vroegindewey, M., Kardys, I., Oemrawsingh, R. M., Cheng, J. M., de Boer, S., et al. (2017). Near-infrared spectroscopy-derived lipid core burden index predicts adverse cardiovascular outcome in patients with coronary artery disease during longterm follow-up. European Heart Journal, 39(4), 295-302. https:// doi.org/10.1093/eurheartj/ehx247.

15. Jansen, K., van Soest, G., \& van der Steen, A. F. (2014). Intravascular photoacoustic imaging: a new tool for vulnerable plaque identification. Ultrasound in Medicine \& Biology, 40(6), 1037-1048. https://doi.org/10.1016/j.ultrasmedbio.2014.01.008.

16. Hui, J., Li, R., Phillips, E. H., Goergen, C. J., Sturek, M., \& Cheng, J.-X. (2016). Bond-selective photoacoustic imaging by converting molecular vibration into acoustic waves. Photoacoustics, 4(1), 1121. https://doi.org/10.1016/j.pacs.2016.01.002.

17. Jansen, K., Wu, M., van der Steen, A. F. W., \& van Soest, G. (2013). Lipid detection in atherosclerotic human coronaries by spectroscopic intravascular photoacoustic imaging. Optics Express, 21(18), 21472-21484. https://doi.org/10.1364/OE.21.021472.

18. Zhang, J., Yang, S., Ji, X., Zhou, Q., \& Xing, D. (2014). Characterization of lipid-rich aortic plaques by intravascular photoacoustic tomography: ex vivo and in vivo validation in a rabbit atherosclerosis model with histologic correlation. Journal of the American College of Cardiology, 64(4), 385-390. https://doi.org/ 10.1016/j.jacc.2014.04.053.

19. VanderLaan, D., Karpiouk, A., Yeager, D., \& Emelianov, S. (2014). System and Integrated Catheter for Real-Time Intravascular Ultrasound and Photoacoustic Imaging. 2014 IEEE International Ultrasonics Symposium, 1591-1594, https://doi.org/10.1109/Ultsym.2014.0394.

20. Wu, M., Springeling, G., Lovrak, M., Mastik, F., Iskander-Rizk, S., Wang, T., et al. (2017). Real-time volumetric lipid imaging in vivo by intravascular photoacoustics at 20 frames per second. Biomedical Optics Express, 8(2), 943-953. https://doi.org/10.1364/boe.8.000943.

21. Hui, J., Cao, Y., Zhang, Y., Kole, A., Wang, P., Yu, G., et al. (2017). Real-time intravascular photoacoustic-ultrasound imaging of lipid-laden plaque in human coronary artery at 16 frames per second. Scientific Reports, 7(1), 1417. https://doi.org/10.1038/s41598-017-01649-9.

22. Cao, Y., Kole, A., Hui, J., Zhang, Y., Mai, J., Alloosh, M., et al. (2018). Fast assessment of lipid content in arteries in vivo by intravascular photoacoustic tomography. Scientific Reports, 8(1), 2400. https://doi.org/10.1038/s41598-018-20881-5.

23. van Soest, G., Marcu, L., Bouma, B. E., \& Regar, E. (2017). Intravascular imaging for characterization of coronary atherosclerosis. Current Opinion in Biomedical Engineering, 3, 1-12. https:// doi.org/10.1016/j.cobme.2017.07.001.

24. Karpiouk, A. B., Wang, B., \& Emelianov, S. Y. (2010). Development of a catheter for combined intravascular ultrasound and photoacoustic imaging. The Review of Scientific Instruments, 81(1), 014901. https://doi.org/10.1063/1.3274197.

25. Wang, B., Su, J. L., Amirian, J., Litovsky, S. H., Smalling, R., \& Emelianov, S. (2010). Detection of lipid in atherosclerotic vessels using ultrasound-guided spectroscopic intravascular photoacoustic imaging. Optics Express, 18(5), 4889-4897. https://doi.org/10. 1364/OE.18.004889.

26. Wang, P., Ma, T., Slipchenko, M. N., Liang, S., Hui, J., Shung, K. K., et al. (2014). High-speed intravascular photoacoustic imaging of lipidladen atherosclerotic plaque enabled by a $2-\mathrm{kHz}$ barium nitrite Raman laser. Scientific Reports, 4, 6889. https://doi.org/10.1038/srep06889.

27. Cao, Y., Hui, J., Kole, A., Wang, P., Yu, Q., Chen, W., et al. (2016). High-sensitivity intravascular photoacoustic imaging of lipid-laden plaque with a collinear catheter design. Scientific Reports, 6, 25236. https://doi.org/10.1038/srep25236.

28. Tanaka, K., \& Sata, M. (2018). Roles of perivascular adipose tissue in the pathogenesis of atherosclerosis. Frontiers in Physiology, 9. https://doi.org/10.3389/fphys.2018.00003.

29. Lee, H. Y., Despres, J. P., \& Koh, K. K. (2013). Perivascular adipose tissue in the pathogenesis of cardiovascular disease. Atherosclerosis, 230(2), 177-184. https://doi.org/10.1016/j. atherosclerosis.2013.07.037.

30. Verhagen, S. N., \& Visseren, F. L. (2011). Perivascular adipose tissue as a cause of atherosclerosis. Atherosclerosis, 214(1), 3-10. https://doi.org/10.1016/j.atherosclerosis.2010.05.034.

31. McKenney-Drake, M. L., Rodenbeck, S. D., Bruning, R. S., Kole, A., Yancey, K. W., Alloosh, M., et al. (2017). Epicardial adipose tissue removal potentiates outward remodeling and arrests coronary atherogenesis. The Annals of Thoracic Surgery, 103(5), 1622-1630. https://doi.org/10.1016/j.athoracsur.2016.11.034.

32. Neeb, Z. P., Edwards, J. M., Alloosh, M., Long, X., Mokelke, E. A., \& Sturek, M. (2010). Metabolic syndrome and coronary artery disease in Ossabaw compared with Yucatan swine. Comparative Medicine, 60(4), 300-315.

33. McKenney-Drake, M. L., Rodenbeck, S. D., Owen, M.K., Schultz, K. A., Alloosh, M., Tune, J. D., et al. (2016). Biphasic alterations in coronary smooth muscle $\mathrm{Ca}(2+)$ regulation in a repeat cross-sectional study of coronary artery disease severity in metabolic syndrome. Atherosclerosis, 249, 1-9. https://doi.org/10.1016/j.atherosclerosis.2016.03.032.

34. Kilic, I. D., Caiazzo, G., Fabris, E., Serdoz, R., Abou-Sherif, S., Madden, S., et al. (2015). Near-infrared spectroscopy-intravascular ultrasound: scientific basis and clinical applications. European Heart Journal Cardiovascular Imaging, 16(12), 1299-1306. https://doi.org/10.1093/ehjci/jev208.

35. Moreno, P. R. (2002). Intimomedial interface damage and adventitial inflammation is increased beneath disrupted atherosclerosis in the aorta: implications for plaque vulnerability. Circulation, 105(21), 2504-2511. https://doi.org/10.1161/01.cir.0000017265.52501.37.

36. Waksman, R., Torguson, R., Spad, M.-A., Garcia-Garcia, H., Ware, J., Wang, R., et al. (2017). The lipid-rich plaque study of vulnerable plaques and vulnerable patients: study design and rationale. American Heart Journal, 192(Supplement C), 98-104. https://doi. org/10.1016/j.ahj.2017.02.010.

37. Bourantas, C. V., Garcia-Garcia, H. M., Torii, R., Zhang, Y. J., Westwood, M., Crake, T., et al. (2016). Vulnerable plaque detection: an unrealistic quest or a feasible objective with a clinical value? Heart, 102(8), 581-589. https://doi.org/10.1136/heartjnl-2015-309060.

38. Hirata, Y., Tabata, M., Kurobe, H., Motoki, T., Akaike, M., Nishio, C., et al. (2011). Coronary atherosclerosis is associated with macrophage polarization in epicardial adipose tissue. Journal of the American College of Cardiology, 58(3), 248-255. https://doi.org/ 10.1016/j.jacc.2011.01.048.

39. Cao, Y., Kole, A., Lan, L., Wang, P., Hui, J., Sturek, M., et al. (2017). Spectral analysis assisted photoacoustic imaging for lipid composition differentiation. Photoacoustics, 7, 12-19. https://doi. org/10.1016/j.pacs.2017.05.002. 